\title{
Correction to: [89Zr]Zr-cetuximab PET/CT as biomarker for cetuximab monotherapy in patients with RAS wild-type advanced colorectal cancer
}

\author{
E. J. van Helden ${ }^{1}$ - S. G. Elias ${ }^{2}$ - S. L. Gerritse ${ }^{1}$ - S. C. van Es $^{3}$ • E. Boon ${ }^{4}$ - M. C. Huisman ${ }^{5}$ • N. C. T. van Grieken ${ }^{6}$. \\ H. Dekker ${ }^{1}$ - G. A. M. S. van Dongen ${ }^{5}$ - D. J. Vugts $^{5}$ - R. Boellaard ${ }^{5}$. C. M. L. van Herpen ${ }^{4}$ - E. G. E. de Vries ${ }^{3}$. \\ W. J. G. Oyen 7,8 - A. H. Brouwers ${ }^{9} \cdot$ H. M. W. Verheul ${ }^{1}$ - O. S. Hoekstra ${ }^{5}$ - C. W. Menke-van der Houven van \\ Oordt ${ }^{1,2,3,4,5,6,7,8,9}$
}

Published online: 13 June 2020

(C) Springer-Verlag GmbH Germany, part of Springer Nature 2020

\section{Correction to: European Journal of Nuclear Medicine and Molecular Imaging (2020) 47:849-859. https://doi.org/10.1007/s00259-019-04555-6}

The Electronic Supplementary Materials are missing in the original article. Kindly check the Electronic Supplementary Materials in this article.

The Publisher regrets this error.

Publisher's note Springer Nature remains neutral with regard to jurisdictional claims in published maps and institutional affiliations.

This article is part of the Topical Collection on Erratum

The online version of the original article can be found at https://doi.org/ 10.1007/s00259-019-04555-6

Electronic supplementary material The online version of this article (https://doi.org/10.1007/s00259-020-04908-6) contains supplementary material, which is available to authorized users.

\section{W. Menke-van der Houven van Oordt} c.menke@amsterdamumc.nl

1 Medical Oncology, Cancer Center Amsterdam, Amsterdam UMC, Location VU Medical Center, Amsterdam, Netherlands

2 Julius Center for Health Sciences and Primary Care, University Medical Center Utrecht, Utrecht University, Utrecht, Netherlands

3 Medical Oncology, University Medical Center Groningen, University of Groningen, Groningen, Netherlands

4 Medical Oncology, Radboud University Medical Center, Nijmegen, Netherlands
5 Radiology and Nuclear Medicine, Cancer Center Amsterdam, Amsterdam UMC, Location VU Medical Center, Amsterdam, Netherlands

6 Pathology, Cancer Center Amsterdam, Amsterdam UMC, Location VU Medical Center, Amsterdam, Netherlands

7 Radiology and Nuclear Medicine, Radboud University Medical Center, Nijmegen, Netherlands

8 The Institute of Cancer Research and The Royal Marsden Hospital, London, UK

9 Nuclear Medicine and Molecular Imaging, University Medical Center Groningen, University of Groningen, Groningen, Netherlands 